\title{
Incidence and Risk Factors for Sac Expansion after Endovascular Aneurysm Repair of Abdominal Aortic Aneurysms
}

\author{
Deokbi Hwang, Hyung-Kee Kim, and Seung Huh
}

Division of Vascular Surgery, Department of Surgery, Kyungpook National University Hospital, School of Medicine, Kyungpook National University, Daegu, Korea

Purpose: This study aimed to examine the sac changes after endovascular aneurysm repair (EVAR) in patients with abdominal aortic aneurysms.

Materials and Methods: We examined the aneurysm sac size initially and regularly after surgery in 157 consecutive patients who underwent EVAR in 2009-2019. Contrast-enhanced computed tomography (CT) scans were used as well as ultrasound images with non-enhanced CT scans in the patients with renal insufficiency. Sac expansion (SE) at 3 years was divided into two categories: progressive SE (PSE) defined as continuous sac enlargement of $\geq 5 \mathrm{~mm}$ on serial follow-up images at 1 and 3 years compared with the initial sac and delayed SE (DSE) defined as re-expansion of $\geq 5 \mathrm{~mm}$ compared with the regressed or stable sac at 1 year. The SE rate at 1 and 3 years and the risk factors for SE at 3 years were analyzed using logistic regression.

Results: During a median follow-up of 32.5 months, nine reinterventions in six patients were performed with open conversion $(n=5)$ and endovascular repair $(n=4)$. At 1 year, 112 patients underwent follow-up imaging. SE and sac regression were noted in $4(3.6 \%)$ and $57(50.9 \%)$ patients, respectively. Of the 64 patients with 3-year follow-up images, $16(25 \%)$ exhibited SE (PSE [n=6] and DSE [n=10]). In the multivariable analysis, the risk factors for overall SE at 3 years were endoleaks at 1 year $(\mathrm{P}=0.006)$ and renal insufficiency $(\mathrm{P}=0.003)$.

Conclusion: During post-EVAR follow-up, patients with any endoleak at 1 year or renal insufficiency must be strictly monitored for SE development.

Key Words: Endovascular aneurysm repair (EVAR), Sac expansion, Risk factors, Renal insufficiency, Endoleak
Received May 10, 2021

Revised September 18, 2021

Accepted October 3, 2021

Published on November 10, 2021

Corresponding author: Hyung-Kee Kim Division of Vascular Surgery, Department of Surgery, Kyungpook National University Hospital, 130 Dongdeok-ro, Jung-gu, Daegu 41944, Korea

Tel: 82-53-420-5605

Fax: 82-53-421-0510

E-mail: hkkim6260@knu.ac.kr http://orcid.org/0000-0002-4436-7424

Copyright (c) 2021 The Korean Society for Vascular Surgery

This is an Open Access article distributed under the terms of the Creative Commons Attribution Non-Commercial License (http://creativecommons.org/licenses/by-nc/4.0) which permits unrestricted non-commercial use, distribution, and reproduction in any medium, provided the original work is properly cited.

Cite this article; Vasc Specialist Int 2021. https://doi.org/10.5758/vsi.210035

\section{INTRODUCTION}

Endovascular aneurysm repair (EVAR) is preferred as the first-choice treatment in fragile patients with abdominal aortic aneurysms (AAAs) requiring treatment. By 2010, the number of EVAR cases reportedly surpassed the number of open repairs in South Korea as well as in the USA [1,2].
However, the 15-year follow-up of EVAR trial 1 demonstrated that the early survival benefit of EVAR is canceled out by the inferior late survival, need for lifelong surveillance, and high rates of reintervention compared with open repair. Further, the increased late aneurysm-related mortality in the EVAR group is mainly attributable to secondary aneurysm sac rupture [3]. 
Sac shrinkage following EVAR is believed to be a surrogate marker of low occurrence of late complications or longer survival [4,5]; however, the incidence of sac expansion (SE) after EVAR is not negligible, and it is generally believed that SE above some degree should be corrected because of the possibility of rupture. The overall SE rate has been reported to be approximately $10 \%$ at 1 year and $40 \%$ at 5 years after EVAR, especially when the anatomy is unfit for instructions for use (IFU) [6,7].

Accordingly, many studies have been conducted regarding the factors influencing the sac changes after EVAR and the relationship between SE and consequences of reintervention or mortality. Many features of endoleaks, increasing age, hostile anatomy, and renal impairment have been presented as possible risk factors for SE after EVAR $[7,8]$. However, there seems to be a lack of robust data to support the factors that can anticipate SE in practice. This study aimed to investigate the sac changes at 1 and 3 years and to identify the predictive factors for SE at 3 years after EVAR.

\section{MATERIALS AND METHODS}

This study was approved by our Institutional Review Board (approval no. 2019-12-001), and given its retrospective design, patient informed consent was not required. Our study included 157 patients who underwent EVAR at a tertiary hospital in South Korea between October 2009 and February 2019. The patients were followed up for a median period of 32.5 (interquartile range, 11.9-55.6) months.

All consecutive patients with both intact and ruptured
AAAs sized $\geq 5 \mathrm{~cm}$ who underwent EVAR were enrolled. Patients who complained of aneurysm-related pain or had a saccular-shaped AAA and received EVAR were included in the study regardless of the aneurysm size. We targeted patients with preoperative and postoperative follow-up images; 112 had images from the first year, while 64 had images from both the first and third years after EVAR. Conversely, patients who received EVAR for iliac artery aneurysms sized $\geq 3.5 \mathrm{~cm}$ with or without small AAAs $(<5 \mathrm{~cm})$ were excluded.

We used the AquariusNET software (TeraRecon Inc., San Mateo, CA, USA) and processed three-dimensional computed tomography (CT) images. The sac diameter, neck length, proximal and distal neck diameters, neck shape, suprarenal and infrarenal angles, distal aortic diameter, and iliac tortuosity were examined in planning EVAR. The aneurysm sac size was individually measured by two vascular surgeons based on a contrast-enhanced CT scan or ultrasound image with a non-enhanced CT scan in the patients with renal impairment. Considering the compatibility with ultrasound, the anteroposterior diameter was preferentially gauged.

The presence of endoleaks was determined by the attending physicians based on $\mathrm{CT}$ angiography or ultrasound images examined by registered vascular technologists. When the patients with renal insufficiency needed frequent follow-up because of SE or suspected endoleaks, contrastenhanced ultrasound (CEUS) was preferred.

As outcomes of interest, we primarily analyzed the size of the aneurysm sac before and at 1 and 3 years after EVAR, suspected cause of SE, and risk factors for overall SE during the follow-up period. The characteristics of the patients and aneurysms and aneurysm-related deaths were

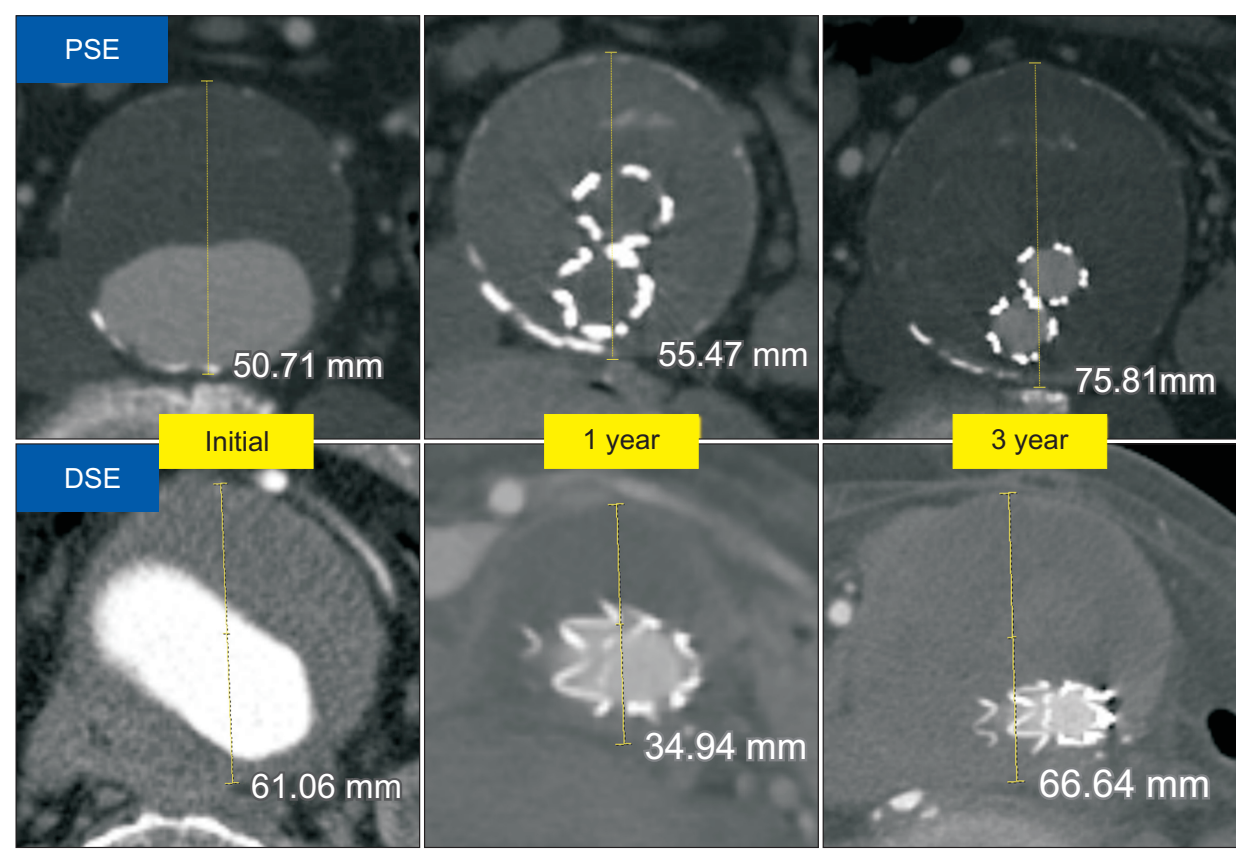

Fig. 1. Examples of progressive sac expansion (PSE) and delayed sac expansion (DSE). PSE group was defined as continuous sac enlargement of $\geq 5 \mathrm{~mm}$ on serial follow-up images and DSE group as re-expansion of aneurysm sac diameter of $\geq 5$ $\mathrm{mm}$ at 3 years compared with the regressed or unchanged aneurysm sac at 1-year image. It was determined whether it had increased or not compared to the previous image, not the initial one. 
also investigated.

We divided the patients with SE at 3 years into two groups: the progressive SE (PSE) group defined as exhibiting continuous sac enlargement of $\geq 5 \mathrm{~mm}$ on serial followup images at 1 and 3 years and the delayed SE (DSE) group defined as presenting re-expansion of the aneurysm sac diameter of $\geq 5 \mathrm{~mm}$ at 3 years compared with the regressed or unchanged aneurysm sac at the 1-year follow-up (Fig. 1). Notably, it was determined whether the size had increased compared with the previous image, not the initial image. The sum of the PSE and DSE groups was considered as the total SE (TSE) group.

\section{1) Definitions}

Renal insufficiency was defined as moderate to severe reduction in the estimated glomerular filtration rate (eGFR, $<60 \mathrm{~mL} / \mathrm{min} / 1.73 \mathrm{~m}^{2}$, stages 3-5).

After completion of endograft placement and before removal of the delivery system, a completion angiogram was performed. Despite various additional procedures to eliminate type 1 or 111 endoleaks, the condition was recorded as a final endoleak when any suspicious type 1 or 111 leakage of contrast medium through the endograft was found.

Overall EVAR suitability was defined when both iliac and standard neck suitabilities were met. A standard (extended) neck IFU was defined as follows: aortic neck length, $\geq 15$ (10) $\mathrm{mm}$; suprarenal angle, $\leq 45^{\circ}$; infrarenal angle, $\leq 60^{\circ}$ (75); and neck diameter, 18 to $32 \mathrm{~mm}$. When an unfavorable aortic neck anatomy, including a short neck, large angulation, and small or large neck diameters, outside each IFU was checked, the patients were categorized into the non-IFU group.

\section{2) Operation details}

When a direct approach to the aneurysm sac was required, we performed laparotomy, approached the retroperitoneal space, and dissected the proximal and distal aorta. Aortotomy was followed by ligation of branches, such as the lumbar artery or inferior mesenteric artery (IMA), omentopexy, or repair of the stent graft. A series of these processes is referred to as "open conversion".

Regarding bleeding through the impaired endograft, we repaired the fabric defect using 6-0 prolene sutures. In this case, we used the expression "suture of the stent graft".

When a type $V$ endoleak (endotension) was identified as the cause of SE and no other leakage or endoprosthesisrelated infection was found, we wrapped the exposed stent graft within the aneurysmal sac with the omentum. The omentum was fixed along the edge of the aneurysmal sac using 3-0 silk sutures. This procedure is called "omentopexy”.

\section{3) Follow-up protocols}

The patients were scheduled to visit the outpatient clinic after discharge at 1, 6, and 12 months after EVAR and every year thereafter. Contrast-enhanced CT scans were obtained at 1 month and every year after EVAR. In the patients without endoleaks at 1 month, a CT scan was obtained annually. In the patients with endoleaks at 1 month, an additional CT scan was obtained 6 months after EVAR. Taking contrast-induced nephropathy into account, non-enhanced CT scans for size measurement and duplex ultrasound images for endoleak evaluation were subsequently examined in the patients with renal impairment but not on dialysis. In the patients with SE, reintervention was considered when they complained of abdominal pain or discomfort or according to the physician's decision considering the patients' condition and sac size.

\section{4) Statistical analysis}

Baseline characteristics, such as age, sex, comorbid conditions, smoking history, and aneurysm characteristics, were included as independent variables. The results were analyzed using IBM SPSS statistics (ver. 20.0; IBM Corp., Armonk, NY, USA). Numeric data with normal distribution were compared using Student t-test and presented as means and standard deviations; categorical variables were compared using the chi-squared test (for adequate-sized samples) or Fisher's exact test (for smaller samples). Logistic regression analyses were performed to identify the factors associated with SE after EVAR, and odds ratios (ORs) with 95\% confidence intervals (Cls) were determined. The factors found to be significant in the univariable analyses were included in the multivariable analyses. Statistical significance for all tests was set at P-values of $<0.05$.

\section{RESULTS}

\section{1) Patient characteristics}

The patient demographics are detailed in Table 1. EVAR was conducted in 157 patients, of whom 129 (82.2\%) were male; the mean age was 73 years. Coronary artery disease (CAD) and cerebrovascular disease (CVD) were found in 57 (36.3\%) and $21(13.4 \%)$ patients, respectively. Twenty-nine percent of the patients were preoperatively diagnosed with renal insufficiency. More than half of the patients had a history of smoking. There were no obvious differences in 
the clinical features among all patients, patients with 1-year follow-up images, and patients with 1- and 3-year followup images.

\section{2) Reintervention owing to sac expansion}

During the median follow-up of 32.5 months, nine reinterventions in six patients were performed owing to SE (Table 2). Two patients underwent multiple reinterventions. In Patient 1, a type lb endoleak was found at 15.7 and 20.3 months postoperatively, and two graft extensions were performed. Patient 2 underwent proximal extension under suspicion of a type la endoleak at approximately 4 years post- operatively; however, the sac continually expanded, and the patient underwent open conversion and suture of the stent graft owing to a type 111 endoleak 6 months later. A month later, he received proximal extension again because of a type la endoleak causing 10-mm SE. In Patient 3, the cause of SE was considered a type 11 endoleak at 37.6 months after EVAR, and lumbar artery ligation was performed. The other three patients underwent open conversion because of SE. Patient 4 with type lb endoleak ensuing 15-mm SE and aneurysm rupture after 40 months underwent hematoma evacuation, end-to-end anastomosis from the left limb to the left common iliac artery, and omentopexy. Since the bleeding site was clear, the endograft was not explanted.

Table 1. Characteristics of patients

\begin{tabular}{lccc}
\hline \multicolumn{1}{c}{ At the time of EVAR } & Total $(\mathrm{n}=157)$ & 1 year follow-up $(\mathrm{n}=112)$ & 3 year follow-up (n=64) \\
\hline Age $(\mathrm{y})$ & $72.9 \pm 6.9$ & $72.5 \pm 6.9$ & $71.2 \pm 7.2$ \\
Sex, male & $129(82.2)$ & $92(82.1)$ & $53(82.8)$ \\
Comorbidities & & & $78(69.6)$ \\
$\quad$ Hypertension & $111(70.7)$ & $8(7.1)$ & $7(10.9)$ \\
Diabetes mellitus & $18(11.5)$ & $42(37.5)$ & $22(34.4)$ \\
CAD & $57(36.3)$ & $10(8.9)$ & $5(7.8)$ \\
CHF & $16(10.2)$ & $31(27.7)$ & $14(21.9)$ \\
Arrhythmia & $41(26.1)$ & $16(14.3)$ & $9(14.1)$ \\
CVD & $21(13.4)$ & $22(19.6)$ & $8(12.5)$ \\
COPD & $37(23.6)$ & $30(26.8)$ & $15(23.4)$ \\
Renal insufficiency & $46(29.3)$ & $56(50.0)$ & $24(37.5)$ \\
Dyslipidemia & $82(52.2)$ & $65(58.0)$ & $34(53.1)$ \\
Smoking history & $92(58.6)$ & $16(14.3)$ & $6(9.4)$ \\
Malignancy & $24(15.3)$ & $98(87.5)$ & $55(85.9)$ \\
Antithrombotics ${ }^{a}$ & $130(82.8)$ & & \\
\hline
\end{tabular}

Values are presented as mean \pm standard deviation or number (\%).

EVAR, endovascular aneurysm repair; CAD, coronary artery disease; CHF, congestive heart failure; CVD, cerebrovascular disease; COPD, chronic obstructive pulmonary disease.

${ }^{a}$ Antithrombotics indicate the incidence of patients who had taken an antiplatelet agent or anticoagulant, irrespective of the number of medications postoperatively.

Table 2. Reinterventions due to sac expansion during follow-up

\begin{tabular}{|c|c|c|c|c|c|c|}
\hline Patient & Possible cause type & Initial sac size $(\mathrm{mm})$ & $\Delta$ Sac size $(\mathrm{mm})$ & Interval (mo) & Method & Procedure \\
\hline \multirow[t]{2}{*}{1} & $\mathrm{lb}$ & 63.4 & 9.1 & 15.7 & Endo & Graft extension \\
\hline & $\mathrm{lb}$ & & 9.0 & 20.3 & Endo & Graft extension \\
\hline \multirow[t]{3}{*}{2} & la & 52.5 & 25.7 & 47.4 & Endo & Proximal extension \\
\hline & III & & 32.9 & 53.2 & $\mathrm{OC}$ & Suture of graft \\
\hline & la & & 42.2 & 54.2 & Endo & Proximal extension \\
\hline 3 & II & 54.4 & 18.3 & 37.6 & $\mathrm{OC}$ & Ligation, LA \\
\hline 4 & $\mathrm{lb}$ & 53.1 & 15.4 & 40.4 & $\mathrm{OC}$ & Anastomosis, E-E, left limb to left CIA, omentopexy \\
\hline 5 & II & 57.1 & 5.3 & 1.8 & $\mathrm{OC}$ & Ligation, LA, IMA \\
\hline 6 & $\mathrm{~V}$ & 53.7 & 8.3 & 6.9 & $\mathrm{OC}$ & Omentopexy \\
\hline
\end{tabular}

Endo, endovascular reintervention; OC, open conversion; LA, lumbar artery; E-E, end to end; CIA, common iliac artery; IMA, inferior mesenteric artery. 
Two other patients underwent ligation of the lumbar artery and IMA for a type 11 endoleak (Patient 5) and omentopexy for a type $\mathrm{V}$ endoleak (Patient 6).

\section{3) Aneurysm-related death}

During the follow-up period, two aneurysm-related deaths occurred. While surveillance of constant sac size, 6-mm SE without definite endoleak was observed at 49 months after EVAR. After 1 month, he was transferred to our emergency room (ER) with loss of consciousness due to excessive bleeding. CT confirmed a ruptured aneurysm due to a type $1 \mathrm{~b}$ endoleak.

The other patient visited the ER with abdominal pain and high fever at 4 years after EVAR. Diagnosed with a graft-related infection, the patient underwent removal of the infected graft and reconstruction with the femoral vein. However, on postoperative day 58, the patient died of hospital-acquired pneumonia.

\section{4) Follow-up images at 1 and 3 years after endovascular aneurysm repair}

One hundred and twelve patients with 1-year followup images and sixty-four patients with both 1- and 3-year

Table 3. Clinical risk factors for total sac enlargement at 3 years

\begin{tabular}{lccr}
\hline \multicolumn{1}{c}{ Variable } & $\begin{array}{c}\text { TSE } \\
(\mathrm{n}=16)\end{array}$ & $\begin{array}{c}\text { Non-TSE } \\
(\mathrm{n}=48)\end{array}$ & P-value \\
\hline Age (y) & $73.1 \pm 7.5$ & $70.9 \pm 7.1$ & 0.312 \\
Sex, male & $14(87.5)$ & $39(81.3)$ & 0.716 \\
Comorbidities & & & \\
Hypertension & $11(68.8)$ & $33(68.8)$ & $>0.999$ \\
Diabetes mellitus & $2(12.5)$ & $5(10.4)$ & $>0.999$ \\
CAD & $6(37.5)$ & $16(33.3)$ & 0.761 \\
CHF & $3(18.8)$ & $2(4.2)$ & 0.095 \\
Arrhythmia & $4(25.0)$ & $10(20.8)$ & 0.736 \\
CVD & $2(12.5)$ & $7(14.6)$ & $>0.999$ \\
COPD & $1(6.3)$ & $7(14.6)$ & 0.667 \\
RI (eGFR <60 mL/min/1.73 $\left.\mathrm{m}^{2}\right)$ & $8(50.0)$ & $7(14.6)$ & 0.005 \\
Dyslipidemia & $7(43.8)$ & $17(35.4)$ & 0.551 \\
Smoking history & $10(62.5)$ & $24(50.0)$ & 0.386 \\
Malignancy & $0(0.0)$ & $6(12.5)$ & 0.323 \\
Antithrombotics & $11(68.8)$ & $43(89.6)$ & 0.039 \\
\hline
\end{tabular}

Values are presented as mean \pm standard deviation or number (\%). TSE, total sac expansion; CAD, coronary artery disease; CHF, congestive heart failure; $C V D$, cerebrovascular disease; COPD, chronic obstructive pulmonary disease; RI, renal insufficiency; eGFR, estimated glomerular filtration rate. follow-up images after EVAR were investigated. In the patients with both 1- and 3-year follow-up images, endoleaks were observed in 14 patients at 1 year, all of whom showed type 11 endoleaks. Endoleaks were found in 13 patients at 3 years, including 12 type 11 endoleaks and 1 type la endoleak. At 1 year, SE of $\geq 5 \mathrm{~mm}$ was noted in four (3.6\%) patients. Two of them underwent open conversions; one patient underwent suture ligation of the lumbar arteries because of a type 11 endoleak, and the other patient underwent omentopexy and proximal fixation for a type $\mathrm{V}$ endoleak. The other two patients were placed under continuous surveillance. Sac regression of $\geq 5 \mathrm{~mm}$ compared with the initial CT finding was found in 57 (50.9\%) patients and a stable sac in 51 (45.5\%) patients.

Three-year follow-up images were obtained in 64 patients and compared with 1-year follow-up images. Sixteen $(25.0 \%)$ of these patients presented with TSE (PSE $[n=6]$ and DSE $[n=10])$. Between the first and third years after EVAR, one patient underwent reintervention owing to SE caused by a type $\mathrm{lb}$ endoleak. Forty-one patients had a stable sac. Sac regression compared with the 1-year followup image finding was observed in seven (10.9\%) patients.

\section{5) Risk factors for total sac expansion}

The proportion of renal insufficiency was significantly higher in the TSE group $(n=16)$ than in the non-TSE group $(n=48)(50.0 \%$ vs. $14.6 \%, P=0.005)$. The proportion of congestive heart failure (CHF) tended to be higher in the TSE group than in the non-TSE group. Regarding antithrombotics, more patients in the non-TSE group took antiplatelet agents or anticoagulants than the TSE group. No significant differences were found in the other clinical factors between the two groups (Table 3).

The anatomic features are detailed in Table 4; therein, no significant difference was found between the two groups. Regarding standard neck IFU suitability, no differences were observed between them. The proportion of patients satisfying each respective neck or iliac suitability, as well as overall suitability, did not also differ. However, the proportion of patients who did not meet the extended neck suitability tended to be higher in the TSE group than in the non-TSE group. Additionally, whether the graft was made of polytetrafluoroethylene (PTFE) did not seem to affect the TSE. The proportion of final endoleaks at the time of surgery tended to be higher in the TSE group than in the non-TSE group ( $25.0 \%$ vs. $8.3 \%, P=0.099$ ). Any endoleak at 1 year $(50.0 \%$ vs. $12.5 \%, \mathrm{P}=0.004)$ and 3 years $(62.5 \%$ vs. $6.3 \%, P<0.001)$ after EVAR occurred more frequently in the TSE group than in the non-TSE group. 
Table 4. Anatomical risk factors for total sac enlargement at 3 years after EVAR

\begin{tabular}{lccr}
\hline \multicolumn{1}{c}{ Variable } & $\begin{array}{c}\text { TSE } \\
(\mathrm{n}=16)\end{array}$ & $\begin{array}{c}\text { Non-TSE } \\
(\mathrm{n}=48)\end{array}$ & P-value \\
\hline Initial diameter $(\mathrm{mm})$ & $55.7 \pm 8.9$ & $57.9 \pm 8.4$ & 0.377 \\
Contained rupture & $1(6.3)$ & $2(4.2)$ & $>0.999$ \\
EVAR suitability & $10(62.5)$ & $27(56.3)$ & 0.661 \\
Neck suitability (standard) & $11(68.8)$ & $36(75.0)$ & 0.745 \\
Neck suitability (extended) & $12(75.0)$ & $44(91.7)$ & 0.099 \\
Neck length (mm) & $30.5 \pm 10.5$ & $33.5 \pm 9.5$ & 0.286 \\
Neck diameter, proximal & $21.2 \pm 2.1$ & $20.7 \pm 2.4$ & 0.401 \\
Neck diameter, distal & $21.5 \pm 2.7$ & $21.4 \pm 2.6$ & 0.960 \\
Neck angulation, suprarenal & $25.6 \pm 24.7$ & $27.4 \pm 20.4$ & 0.782 \\
Neck angulation, infrarenal & $49.3 \pm 25.1$ & $43.0 \pm 22.0$ & 0.345 \\
Conical neck & $1(6.3)$ & $7(14.6)$ & 0.667 \\
Aortic tortuosity & $1.11 \pm 0.08$ & $1.10 \pm 0.07$ & 0.688 \\
Iliac suitability & $13(81.3)$ & $35(72.9)$ & 0.740 \\
CIA length, right & $37.6 \pm 14.3$ & $42.0 \pm 19.4$ & 0.406 \\
CIA length, left & $44.9 \pm 14.1$ & $44.7 \pm 20.1$ & 0.970 \\
CIA aneurysm & $0(0.0)$ & $3(6.3)$ & 0.567 \\
PTFE graft & $11(68.8)$ & $38(79.2)$ & 0.498 \\
Final endoleak & $4(25.0)$ & $4(8.3)$ & 0.099 \\
Endoleak at 1-year & $8(50.0)$ & $6(12.5)$ & 0.004 \\
Endoleak at 3-year & $10(62.5)$ & $3(6.3)$ & $<0.001$ \\
\hline
\end{tabular}

Values are presented as mean \pm standard deviation or number (\%). EVAR, endovascular aneurysm repair; TSE, total sac expansion; CIA, common iliac artery; PTFE, polytetrafluoroethylene.
6) Multivariable analysis for the influencing factors of total sac expansion at 3 years

In the univariable analysis, renal insufficiency (OR: 6.53, 95\% Cl: 1.79-23.81, $\mathrm{P}=0.004)$ and the presence of an endoleak at 1 year (OR: 7.00, 95\% Cl: 1.91-25.71, $\mathrm{P}=0.003$ ) were significantly associated with the TSE at 3 years after EVAR. Furthermore, the analysis demonstrated that CHF, extended neck suitability, and the intraoperative presence of a final endoleak were associated with the TSE; however, the difference was not significant (Table 5). Antithrombotics seemed to inversely affect the TSE (OR: $0.21,95 \% \mathrm{Cl}$ : 0.047-0.89, $\mathrm{P}=0.035$ ). However, in the multivariable analysis, only renal insufficiency (adjusted OR [aOR]: 16.85, 95\% Cl: 2.58-110.03, $\mathrm{P}=0.003$ ) or an endoleak at 1 year (aOR: 13.64, 95\% Cl: 2.09-88.98, $\mathrm{P}=0.006$; Table 5) was significantly associated with the TSE.

\section{DISCUSSION}

Despite its position as an attractive alternative to open repair, EVAR has constantly been associated with the issue of long-term durability and surveillance. Sac behavior after EVAR has been considered an indicator of success or failure. In this study, the sac changes at 1 and 3 years and risk factors for TSE at 3 years after EVAR were investigated; the incidence of SE at 1 year after EVAR was lower in our study than in a previous literature [6]. However, based on the 3-year follow-up results, considerable cases of delayed expansion occurred in the patients with no sac change or

Table 5. Multivariable analysis for influencing factors of total sac expansion at 3 years after EVAR

\begin{tabular}{|c|c|c|c|c|}
\hline \multirow{2}{*}{ Variable } & \multicolumn{2}{|c|}{ Univariable analysis } & \multicolumn{2}{|c|}{ Multivariable analysis } \\
\hline & OR (95\% Cl) & P-value & Adjusted OR (95\% Cl) & P-value \\
\hline Age & $1.06(0.97-1.15)$ & 0.217 & & \\
\hline Sex, Female & $0.62(0.12-3.22)$ & 0.569 & & \\
\hline CAD & $1.20(0.37-3.89)$ & 0.761 & & \\
\hline $\mathrm{CHF}$ & $5.31(0.80-35.21)$ & 0.084 & $1.73(0.24-93.32)$ & 0.307 \\
\hline $\mathrm{RI}\left(\mathrm{eGFR}<60 \mathrm{~mL} / \mathrm{min} / 1.73 \mathrm{~m}^{2}\right)$ & $6.53(1.79-23.81)$ & 0.004 & $16.85(2.58-110.03)$ & 0.003 \\
\hline CVD & $0.84(0.16-4.51)$ & 0.836 & & \\
\hline Dyslipidemia & $1.42(0.45-4.49)$ & 0.552 & & \\
\hline Smoking history & $1.67(0.52-5.31)$ & 0.388 & & \\
\hline Size (initial) & $1.03(0.97-1.10)$ & 0.376 & & \\
\hline PTFE & $0.58(0.16-2.05)$ & 0.397 & & \\
\hline Neck suitability (extended) & $0.27(0.06-1.25)$ & 0.095 & & \\
\hline Endoleak at 1 year & $7.00(1.91-25.71)$ & 0.003 & 13.64 (2.09-88.98) & 0.006 \\
\hline Final endoleak & $3.91(0.96-15.94)$ & 0.057 & $3.25(0.46-22.77)$ & 0.236 \\
\hline Antithrombotics & $0.21(0.047-0.89)$ & 0.035 & & \\
\hline
\end{tabular}

EVAR, endovascular aneurysm repair; $\mathrm{OR}$, odds ratio; $\mathrm{Cl}$, confidence interval; $\mathrm{CAD}$, coronary artery disease; $\mathrm{CHF}$, congestive heart failure; $\mathrm{RI}$, renal insufficiency; eGFR, estimated glomerular filtration rate; CVD, cerebrovascular disease; PTFE, polytetrafluoroethylene. 
regression at 1 year after EVAR. Of the 16 patients with TSE, 10 (62.5\%) showed a stable or regressed sac at 1 year. This result indicates that sac shrinkage or stabilization can be achieved in the early phase, within at least 1 year after EVAR; however, patients should be continuously monitored because of the non-negligible probability of DSE.

Several factors, including younger age, smoking, and anatomical characteristics, such as a larger aneurysm diameter, a long and small neck, a higher proportion of thrombus, and less neck calcification, have been reported to affect sac reduction, although severe neck angulation, patent aortic side branches and their persistent endoleaks, and renal impairment are considered causes of SE [8-11]. There are many reports on the association of persistent endoleaks or inappropriate anatomical features with SE [7,12-17].

In this study, we compared the 3-year follow-up images of 64 patients with their 1-year follow-up images, and $16(25.0 \%)$ patients demonstrated SE over $5 \mathrm{~mm}$. Among them, an endoleak was noted in 8 patients at 1 year and 10 patients at 3 years. The most suspected type of an endoleak was type 11 ( $n=7$ at 1 year and $n=9$ at 3 years). The possibility of SE was higher in the patients with type 1 or 111 endoleaks than in the patients with type 11 endoleaks because of the large pressurizing effect of the direct flow from the aorta. In contrast, it is still controversial whether type 11 endoleaks are generally considered benign. Some investigators have argued that the overall all-cause and aneurysmrelated mortalities are unaffected by the presence of a type 11 endoleak. According to them, patients who were simply observed for type 11 endoleak-associated sac growth had aneurysm-related outcomes similar to those of patients who underwent reintervention $[18,19]$. However, type 11 endoleaks were the main type observed in our patients with TSE at 3 years, and it can be estimated that an untreated type 11 endoleak causes an increase in the intrasac pressure and consequent SE, which in turn produces a crack between the device and aortic wall, other types of endoleaks, and resultant sac rupture at the end. According to one report, type 1 or 111 endoleaks were newly discovered at a delayed timepoint in approximately 20\% of patients with type 11 endoleaks [18]. In fact, our data indicated that two of the nine patients with type 11 endoleaks accompanied with SE at 3 years received reintervention because of newly discovered type 1 endoleaks during follow-up. Given these results, the treatment of type 11 endoleaks at 1 year seems to be of considerable importance. Regarding the efficacy and safety of embolization into the sac or branch vessels, favorable results in preventing type 11 endoleaks or reintervention have been reported [20-25]. Therefore, embolization before or during EVAR can be considered for the prevention of type 11 endoleaks and their associated complications, especially in patients with a high risk of endoleaks.

Age, sex, other vascular diseases (CAD or CVD), smoking, and dyslipidemia, which were previously deemed meaningful, appeared to be nonsignificant; however, this finding is rather unreliable because of the small number of patients included. However, considering the significant results of the multivariable analysis, if patients with renal insufficiency must undergo EVAR, special attention should be paid to SE. It is well known that patients with CKD have a poor prognosis after open repair for AAAs [26-28]. However, because of the life-threatening course of AAAs when untreated, patients and physicians have no choice but to proceed with treatment. Because of the vulnerability of patients, EVAR tends to be preferred over open repair, notwithstanding the possibility of future reintervention. Nonetheless, both choices must be individually determined for each patient.

In this study, the patients with renal insufficiency accounted for $29.3 \%$ of all patients. As an influencing factor for sac behavior, renal impairment has not been well investigated. Patients with renal impairment usually have other cardio-cerebrovascular diseases that require medication. Warfarin or antiplatelet agents are known to induce bleeding tendencies, and in patients taking these drugs, a high risk of endoleaks has been reported [29]. In our study, most patients (82.8\%) were taking at least one antiplatelet or anticoagulant agent. Of the 16 patients with TSE, 11 received a single antithrombotic agent. Significantly more patients in the non-TSE group were prescribed antiplatelet agents than those in the TSE group. However, this should not be interpreted as a protective effect of the medication against SE. Antiplatelets are usually prescribed after EVAR. In several patients with definite endoleaks or suspicious findings on a completion angiogram, this medication was not prescribed. In fact, final endoleaks appeared to affect the prescription of antithrombotics (OR: 0.153, 95\% Cl: $0.032-0.732, \mathrm{P}=0.019$ ). Based on our study findings, it was difficult to estimate the impact of antithrombotics on SE.

Of the 16 patients in the TSE group, an endoleak was not detected in eight (50.0\%) patients at 1 year and six (37.5\%) patients at 3 years, which suggests the possibility of endotension. Intriguingly, half of these patients showed renal insufficiency at 1 year $(n=4)$ and 3 years $(n=3)$. Additionally, in 51 patients with both 1- and 3-year followup images and without any endoleak at 3 years, only renal insufficiency showed a significant association with the TSE (aOR: 7.783, 95\% Cl: 1.037-58.408, $\mathrm{P}=0.046$ ) after adjusting for other factors. Neither medication $(\mathrm{P}=0.568)$ nor any endoleak at 1 year $(\mathrm{P}=0.420)$ was associated with the TSE. Extensive research is needed to determine whether endotension occurs more frequently in patients with renal insufficiency. 
As for Patient 2, proximal extension could be performed twice without chimney or snorkel techniques because the patient had been diagnosed with CKD and started dialysis 2 years after EVAR. Regarding the treatment of type 11 endoleaks, suture of the stent graft was proven to be insufficient given the subsequent reintervention in a short period. Notably, replacing the entire graft with a new material would be more desirable to prevent endoleaks, especially if the landing zone is expected to be insufficient for extension of the stent graft.

According to this study, the graft material (PTFE) had no influence on the TSE (Table 4). Regarding the device brand, a total of seven kinds (Endurant [Medtronic Cardiovascular, SantaRosa, CA, USA] [n=23], Endurant lls [Medtronic Cardiovascular] [n=28], Excluder [WL Gore and Associates, Flagstaff, AZ, USA] [n=61], C3 [WL Gore and Associates] [n=24], InCraft [Cordis Corp., Bridgewater, NJ, USA] [n=3], AFX [Endologix, Irvine, CA, USA] [n=9], and Zenith Flex [Cook Medical, Bloomington, IN, USA] [n=9]) of stent grafts were used. The number of cases was too small to assess its association with SE. However, in 64 patients with both 1and 3-year follow-up images, only four types of endografts were used, and there was no significant association found between the device brand and SE or any endoleak at 1 and 3 years. Similarly, when Excluder and C3 were classified as Gore and Endurant and Endurant lls as Medtronic, no significant correlation was observed (Supplementary Table 1).

Our study has several limitations. Three-year followup images were not available for 45 patients owing to the short follow-up duration. Thirty-two patients died within 3 years after surgery; 14 were lost to follow-up; and only a limited number of follow-up images (1-year follow-up images in 71.3\% of the patients and 3-year follow-up images in $40.8 \%$ ) were available, which seemed to have decreased the statistical power. However, the patients without 1-year follow-up images had mostly undergone recent surgeries, and most patients who were not followed up had already died, which probably suggests our appropriate patient selection for EVAR. Furthermore, because most patients with CKD (73.9\%) were classified under stage 3, it was difficult to evaluate the difference according to the extent of renal impairment. In addition, there was no consistency in the size or endoleak measurement tools among all patients. However, in the patients with renal insufficiency, nonenhanced CT scans were combined with ultrasound images for evaluation, and in most patients, the same modality was consistently applied for the 1- and 3-year evaluations. Our study included patients with ruptured AAAs. It may be argued that the clinical situation of EVAR for ruptured AAAs can be completely different from that of elective EVAR. However, comparative research of sac changes between ruptured and intact AAAs after EVAR has rarely been performed. One recent study showed that there was no difference in the sac behavior between ruptured and intact AAAs treated with EVAR from postoperative 1 year [30]. Based on this finding, we included patients with ruptured AAAs to reflect real-world data. However, the number of ruptured AAAs $(n=8)$ was too small to assess the interrelationship between rupture and any endoleak or SE. In addition, the preoperative patency of the aortic branch vessels was not investigated, and the eGFR during follow-up was not evaluated because the patients under ultrasound surveillance did not undergo blood tests. Lastly, 44.4\% and 57.1\% of the patients with an endoleak at 1 and 3 years received ultrasound surveillance, respectively, as well as $75.0 \%$ of the patients $(n=12)$ in the TSE group. In practice, CEUS has often been used to detect endoleaks after EVAR because of its high sensitivity and no risk of nephrotoxicity or radiation exposure. However, it may be argued that a more ideal surveillance system should be developed.

\section{CONCLUSION}

A significant proportion of patients demonstrated DSE at the 3-year imaging follow-up compared with the 1-year imaging follow-up; therefore, strict monitoring and surveillance are important for patients with any endoleak at 1 year or renal insufficiency, as SE may occur even after initial sac shrinkage or stabilization.

\section{CONFLICTS OF INTEREST}

The authors have nothing to disclose.

\section{ORCID}

\author{
Deokbi Hwang \\ https://orcid.org/0000-0003-0050-6434 \\ Hyung-Kee Kim \\ https://orcid.org/0000-0002-4436-7424 \\ Seung Huh \\ https://orcid.org/0000-0002-0275-4960
}

\section{AUTHOR CONTRIBUTIONS}

Conception and design: HKK, SH. Analysis and interpretation: DH, HKK. Data collection: DH, HKK. Writing the article: DH, HKK. Critical revision of the article: HKK, SH. Final approval of the article: all authors. Statistical analysis: DH, HKK. Obtained funding: none. Overall responsibility: HKK. 
1) Joh JH, Park YY, Cho SS, Park HC. National trends for open and endovascular repair of aneurysms in Korea: 2004-2013. Exp Ther Med 2016;12:3333-3338.

2) Dua A, Kuy S, Lee CJ, Upchurch GR Jr, Desai SS. Epidemiology of aortic aneurysm repair in the United States from 2000 to 2010. J Vasc Surg 2014;59:1512-1517.

3) Patel R, Sweeting MJ, Powell JT, Greenhalgh RM; EVAR trial investigators. Endovascular versus open repair of abdominal aortic aneurysm in 15-years' follow-up of the UK endovascular aneurysm repair trial 1 (EVAR trial 1): a randomised controlled trial. Lancet 2016;388:2366-2374.

4) Fujimura N, Matsubara K, Takahara M, Harada H, Asami A, Shibutani S, et al. Early sac shrinkage is a good surrogate marker of durable success after endovascular aneurysm repair in Japanese patients. J Vasc Surg 2018;67:1410-1418.e1.

5) Houbballah R, Majewski M, Becquemin JP. Significant sac retraction after endovascular aneurysm repair is a robust indicator of durable treatment success. J Vasc Surg 2010;52:878-883.

6) Deery SE, Ergul EA, Schermerhorn ML, Siracuse JJ, Schanzer A, Goodney PP, et al. Aneurysm sac expansion is independently associated with late mortality in patients treated with endovascular aneurysm repair. J Vasc Surg 2018;67:157-164.

7) Schanzer A, Greenberg RK, Hevelone N, Robinson WP, Eslami MH, Goldberg RJ, et al. Predictors of abdominal aortic aneurysm sac enlargement after endovascular repair. Circulation 2011;123:2848-2855.

8) Lalys F, Daoudal A, Gindre J, Göksu C, Lucas A, Kaladji A. Influencing factors of sac shrinkage after endovascular aneurysm repair. J Vasc Surg 2017;65:1830-1838.
9) Soler RJ, Bartoli MA, Mancini J, Lerussi G, Thevenin B, Sarlon-Bartoli G, et al. Aneurysm sac shrinkage after endovascular repair: predictive factors and long-term follow-up. Ann Vasc Surg 2015;29:770-779.

10) Sadek M, Dexter DJ, Rockman CB, Hoang H, Mussa FF, Cayne NS, et al. Preoperative relative abdominal aortic aneurysm thrombus burden predicts endoleak and sac enlargement after endovascular anerysm repair. Ann Vasc Surg 2013;27:1036-1041.

11) Kaladji A, Cardon A, Abouliatim 1 , Campillo-Gimenez B, Heautot JF, Verhoye JP. Preoperative predictive factors of aneurysmal regression using the reporting standards for endovascular aortic aneurysm repair. J Vasc Surg 2012;55:1287-1295.

12) Higashiura W, Greenberg RK, Katz E, Geiger L, Bathurst S. Predictive factors, morphologic effects, and proposed treatment paradigm for type 11 endoleaks after repair of infrarenal abdominal aortic aneurysms. J Vasc Interv Radiol 2007;18:975-981.

13) Karthikesalingam A, Thrumurthy SG, Jackson D, Choke E, Sayers RD, Loftus $\mathrm{IM}$, et al. Current evidence is insufficient to define an optimal threshold for intervention in isolated type 11 endoleak after endovascular aneurysm repair. J Endovasc Ther 2012;19:200208.

14) Nolz R, Teufelsbauer H, Asenbaum U, Beitzke D, Funovics M, Wibmer A, et al. Type 11 endoleaks after endovascular repair of abdominal aortic aneurysms: fate of the aneurysm sac and neck changes during long-term follow-up. J Endovasc Ther 2012;19:193199.

15) El Batti S, Cochennec F, RoudotThoraval F, Becquemin JP. Type 11 endoleaks after endovascular repair of abdominal aortic aneurysm are not always a benign condition. J Vasc Surg
2013;57:1291-1297.

16) Kray J, Kirk S, Franko J, Chew DK. Role of type 11 endoleak in sac regression after endovascular repair of infrarenal abdominal aortic aneurysms. J Vasc Surg 2015;61:869-874.

17) Nakai M, Ikoma A, Sato H, Sato M, Nishimura Y, Okamura Y. Risk factors associated with late aneurysmal sac expansion after endovascular abdominal aortic aneurysm repair. Diagn lnterv Radiol 2015;21:195-201.

18) Walker J, Tucker LY, Goodney P, Candell L, Hua H, Okuhn S, et al. Type 11 endoleak with or without intervention after endovascular aortic aneurysm repair does not change aneurysmrelated outcomes despite sac growth. J Vasc Surg 2015;62:551-561.

19) Sidloff DA, Gokani V, Stather PW, Choke E, Bown MJ, Sayers RD. Type 11 endoleak: conservative management is a safe strategy. Eur J Vasc Endovasc Surg 2014;48:391-399.

20) Piazza M, Squizzato F, Zavatta M, Menegolo M, Ricotta JJ 2nd, Lepidi $S$, et al. Outcomes of endovascular aneurysm repair with contemporary volume-dependent sac embolization in patients at risk for type 11 endoleak. J Vasc Surg 2016;63:32-38.

21) Pilon F, Tosato F, Danieli D, Campanile F, Zaramella M, Milite D. Intrasac fibrin glue injection after platinum coils placement: the efficacy of a simple intraoperative procedure in preventing type 11 endoleak after endovascular aneurysm repair. Interact Cardiovasc Thorac Surg 2010;11:78-82.

22) Zanchetta M, Faresin F, Pedon L, Ronsivalle S. Intraoperative intrasac thrombin injection to prevent type 11 endoleak after endovascular abdominal aortic aneurysm repair. J Endovasc Ther 2007;14:176-183.

23) Nevala T, Biancari F, Manninen H, Matsi P, Mäkinen K, Ylönen K, et al. Inferior mesenteric artery emboliza- 
tion before endovascular repair of an abdominal aortic aneurysm: effect on type 11 endoleak and aneurysm shrinkage. J Vasc Interv Radiol 2010;21:181185.

24) Ward TJ, Cohen S, Fischman AM, Kim E, Nowakowski FS, Ellozy SH, et al. Preoperative inferior mesenteric artery embolization before endovascular aneurysm repair: decreased incidence of type 11 endoleak and aneurysm sac enlargement with 24-month followup. J Vasc Interv Radiol 2013;24:4955.

25) Manunga JM, Cragg A, Garberich R, Urbach JA, Skeik N, Alexander J, et al. Preoperative inferior mesenteric artery embolization: a valid method to re- duce the rate of type 11 endoleak after EVAR? Ann Vasc Surg 2017;39:40-47.

26) Nguyen BN, Neville RF, Rahbar R, Amdur R, Sidawy AN. Comparison of outcomes for open abdominal aortic aneurysm repair and endovascular repair in patients with chronic renal insufficiency. Ann Surg 2013;258:394399.

27) Patel Vl, Lancaster RT, Mukhopadhyay $\mathrm{S}$, Aranson NJ, Conrad MF, LaMuraglia $\mathrm{GM}$, et al. Impact of chronic kidney disease on outcomes after abdominal aortic aneurysm repair. J Vasc Surg 2012;56:1206-1213.

28) Walsh SR, Tang T, Sadat U, Varty K, Boyle JR, Gaunt ME. Preoperative glomerular filtration rate and outcome following open abdominal aortic aneurysm repair. Vasc Endovascular Surg 2007;41:225-229.

29) Aoki A, Suezawa T, Sangawa K, Tago M. Effect of type 11 endoleaks and antiplatelet therapy on abdominal aortic aneurysm shrinkage after endovascular repair. J Vasc Surg 2011;54:947951.

30) Oliveira-Pinto J, Soares-Ferreira R, Oliveira NFG, Bouwens E, Bastos Gonçalves FM, Hoeks S, et al. Aneurysm volumes after endovascular repair of ruptured vs intact aortic aneurysms: a retrospective observational study. J Endovasc Ther 2021;28:146-156. 